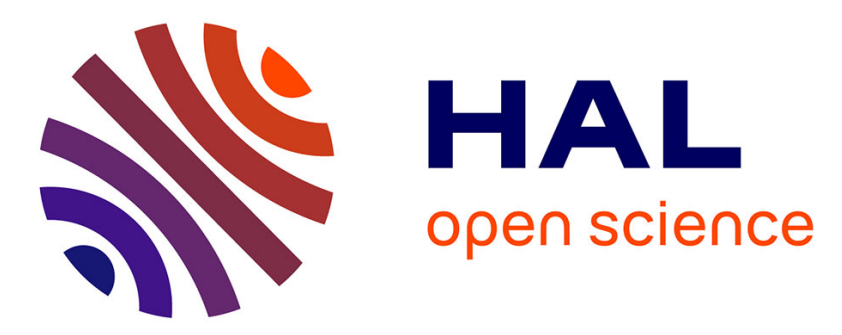

\title{
L'incertitude liée aux avalanches dans les Alpes du Nord : identifications et implications pour la gestion
}

Amandine Moulin, Patrick Pigeon

\section{To cite this version:}

Amandine Moulin, Patrick Pigeon. L'incertitude liée aux avalanches dans les Alpes du Nord: identifications et implications pour la gestion. Neige et glace de montagne: Reconstitution, dynamiques, pratiques., Collection EDYTEM - Cahiers de Géographie, n8, pp.239-248, 2009. halsde-00404064

\section{HAL Id: halsde-00404064 https://hal.science/halsde-00404064}

Submitted on 6 Apr 2011

HAL is a multi-disciplinary open access archive for the deposit and dissemination of scientific research documents, whether they are published or not. The documents may come from teaching and research institutions in France or abroad, or from public or private research centers.
L'archive ouverte pluridisciplinaire HAL, est destinée au dépôt et à la diffusion de documents scientifiques de niveau recherche, publiés ou non, émanant des établissements d'enseignement et de recherche français ou étrangers, des laboratoires publics ou privés. 


\section{Collection EDYTEM}

Numéro 8 - Année 2009

Cahiers de

Géographie

\section{NEIGE et GLACE de MONTAGNE}

Reconstitution, dynamique, pratiques

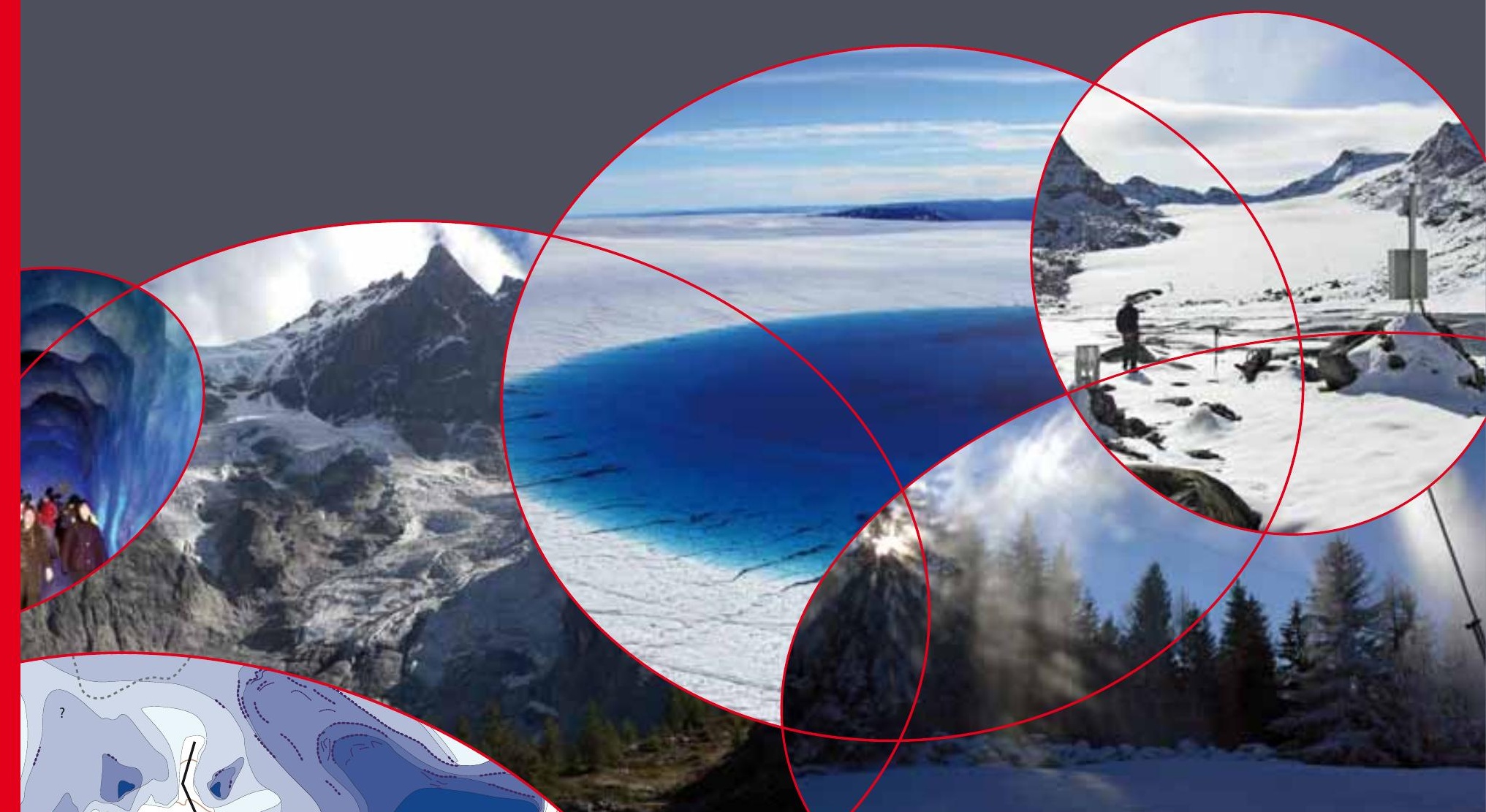





\title{
L'INCERTITUDE LIÉE AUX AVALANCHES DANS LES ALPES DU NORD
}

\section{IDENTIFICATIONS ET IMPLICATIONS POUR LA GESTION}

\author{
SNOWSLIDES RELATED UNCERTAINTIES IN THE NORTHERN FRENCH ALPS: \\ IDENTIFICATION AND MANAGEMENT ISSUES \\ AMANDine MOULIN ${ }^{1}$, PATRICK PIGEON ${ }^{2}$ \\ ${ }^{1}$ Cabinet Uguet, Haute-Savoie. \\ ${ }^{2}$ Laboratoire EDYTEM, Université de Savoie/CNRS, Campus scientifique, F 73376 Le Bourget-du-Lac cedex. \\ Contact : a.moulin@uguet.fr
}

\begin{abstract}
RÉSUMÉ
L'article présente comment l'incertitude liée aux avalanches peut être identifiée dans les Alpes du Nord, tout particulièrement à partir de février 1999. Il expose les facteurs principaux qui permettent d'expliquer une telle évolution. Puis les solutions qui sont recherchées afin de réduire la part de l'incertitude dans les politiques qui cherchent à gérer les risques d'avalanche, sans pouvoir totalement l'éliminer.
\end{abstract}

Mots-CLÉS : AVALANCHES, RISQUES, INCERTITUDE, TRAVAUX DE CORRECTION, URBANISATION, SÉCURITÉ CIVILE, ALPES DU NORD, FRANCE, SUISSE, VAL D'AOSTE.

\begin{abstract}
This paper depicts how uncertainty can be identified today, in relation with snowslide risk management in the Northern Alps, and especially since February 1999 snow slides. It explains the ground causes and trends underlying this evolution. And it gives insights on how uncertainty can be reduced, without allowing to get rid of it.
\end{abstract}

KEYWORDS: SNOWSLIDES, RISKS, UNCERTAINTY, CORRECTIVE WORKS, URBANIZATION, CIVIL SECURITY MANAGEMENT, NORTHERN ALPS, FRANCE, SWITZERLAND, VAL D'Aoste. 


\section{INTRODUCTION}

Cet article propose d'identifier les facteurs d'incertitude qui peuvent remettre en question le risque d'avalanche et rendre plus difficile sa gestion. Il résulte des efforts de réflexion que mènent une ingénieuregéographe ayant la pratique de la gestion du risque (A. Moulin) et un chercheur sur les risques-l'incertitude (P. Pigeon). Pourquoi s'intéresser à ce sujet spécifique ? Dans un premier temps, on pourrait croire que le risque d'avalanche ne devrait pas poser de problème particulier, surtout dans les Alpes. Comme tout risque, il doit reposer sur une connaissance statistique des événements passés, et il concerne ici une montagne très anthropisée sur la durée historique. De plus, cette connaissance a été mobilisée par des organismes spécialisés (CEMAGREF, Centre d'Etude de la Neige, Institut Fédéral pour l'Etude de la Neige et des Avalanches) et de nombreux chercheurs tout au long du $\mathrm{XX}^{\mathrm{e} m e}$ siècle. Ils sont organisés en réseau (ANENA). Ces institutions et ces recherches répondaient notamment à une demande sociale et politique visant à cartographier le risque d'avalanche. Elles ont été remobilisées tout spécialement à la suite des avalanches de février 1999, qui concernèrent surtout les Savoie, la Suisse et le Val d'Aoste, mais aussi le Vorarlberg autrichien, à Galtür. Ce dont témoigne la bibliographie.
Précisément, les avalanches de février 1999 ont fait reconnaître les limites des efforts de gestion qui s'appuient sur la connaissance des événements passés et leur mobilisation à des fins de prédiction/prévention. La première partie de l'article identifie l'incertitude liée aux avalanches. Elle nous incite à nous demander pourquoi l'incertitude est de plus en plus reconnue aujourd'hui. La tendance ne vaut pas que pour les avalanches. Elle concerne aussi d'autres types de risques, comme les inondations (Domenichini, 2008 ; Dupont et Pigeon, 2008). L'évolution est en relation étroite avec les mutations intensifiées des peuplements humains, comme, possiblement, avec les mutations environnementales associées. Leur caractère historiquement inédit gêne la gestion préventive. C'est ce que présente la deuxième partie. Se pose alors une question majeure, actuelle : comment faire pour réduire l'incertitude de telle manière que les efforts de gestion soient politiquement et socialement acceptables, tout en admettant les limites de la prévision? La troisième partie de l'article propose quelques pistes, en remobilisant les efforts de réflexion menés lors des retours d'expérience récents. Ce fut tout particulièrement le cas en Suisse et en France, comme au Val d'Aoste, et en Autriche après les avalanches de 1999.

\section{I - QU'EST-CE QUE L'INCERTITUDE LIÉE AUX AVALANCHES?}

C'est au cours du $\mathrm{XX}^{\mathrm{e}}$ siècle qu'a été formalisée la distinction entre risque et incertitude, comme le développent Godard et al. (2002). Selon ces auteurs, «il s'agit de risque si l'incertitude est définie par une distribution de probabilités objectives. On dit de probabilités qu'elles sont objectives si elles sont établies à partir d'une information statistique, que donnent par exemple en médecine des enquêtes épidémiologiques». La distinction avec l'incertitude est alors justifiée : «l'incertitude ne peut pas être cernée par une distribution de probabilités objectives». Cette distinction entre risque et incertitude est issue des travaux de Keynes (1921) et de Knight (1921), selon Dupuy (2002). Elle est reprise dans des rapports qui présentent les programmes de recherche sur les avalanches : «la notion de risque est associée à un avenir incertain» (TR ALPRISK, 2004). On pose alors que la quantification, la mesure du risque, réduisent l'incertitude, et que, à l'inverse, le déficit ou les difficultés de quantification l'augmentent.

Comment identifie-t-on les limites de la quantification, et du risque ? En nous appuyant sur Godard et $a l$. (2002), nous développons deux pistes en lien direct avec les avalanches. L'incertitude peut provenir du fait que les «probabilités qui sont avancées sont jugées peu crédibles», mais aussi de l'«impossibilité d'établir des probabilités face à une situation nouvelle».

\section{1 - Malgré les bases de données existantes, on identifie des «probabilités jugées non crédibles»}

\section{a - La base de données EPA devrait permettre les calculs de probabilité crédibles}

En Savoie, les premiers relevés des avalanches ont été organisés dans le cadre des politiques de Restauration des Terrains en Montagne sur une centaine de sites, dans l'objectif d'une étude statistique. Cette enquête a été généralisée en Savoie et HauteSavoie dès 1900 , et systématiquement menée à partir de 1920 sous l'impulsion de l'Inspecteur général des Eaux et Forêts Mougin (Strazzeri, Manche, 1998). Au total, 4200 sites sont observés, qui concernent 525 communes et 11 départements. La saisie et le contrôle des données sont effectués par le CEMAGREF. La base de données informatisée comprend plus de 75000 événements. En moyenne par an, 1100 événements sont relevés. L'Enquête Permanente sur les Avalanches (EPA) constitue ainsi un inventaire des dates et des caractéristiques des événements survenus.

L'avalanche du 10 février 1970 à Val d'Isère tuant 39 adolescents d'un centre UCPA toucha l'opinion publique. En réponse, le gouvernement réagit en met- 
tant en place une carte qui visait à inventorier tous les sites d'avalanche. La Carte de Localisation des Phénomènes (terme remplacé par Probable actuellement) d'Avalanche (CLPA) était alors née. Sur fond de carte au 1/25000, les emprises maximales des avalanches connues sont reportées. La Figure 1 en fournit une illustration.

L'EPA et la CLPA constituent donc une base très précieuse en raison du caractère méthodique et précis des renseignements inventoriés, datés et géoréférencés.

Pourtant, les efforts méthodologiques n'empêchent pas des anomalies, comme des distorsions d'informations. L'hétérogénéité de la base de données est alors reconnue. Il devient plus délicat de l'utiliser pour mesurer le risque.

\section{b - Plusieurs facteurs réduisent la crédibilité du calcul de probabilités}

Ils sont essentiellement liés à des anomalies de témoignages, voire aux conditionnements socio-politiques de témoignages passés. Ils rendent la base de données plus hétérogène que ce que laisserait croire la première approche. Ce problème n'est pas spécifique aux avalanches, il se retrouve avec les inondations (Domenichini, 2008).
Par exemple, certaines données EPA sont jugées non crédibles, car l'altitude d'arrivée de l'avalanche est inférieure au point bas du couloir. Comment interpréter cette donnée, faut-il prendre le point bas du couloir ? Concernant les altitudes de départ des avalanches en Savoie, près de 33\% des relevés présentent des altitudes non renseignées ou fausses (GARCIA, 2002).

De plus, les documents anciens sont des constatations de dommages faites à la demande des populations de montagne dans le but d'obtenir des dégrèvements d'impôts. En effet, c'est par le biais de ce type de mesures que la monarchie d'Ancien Régime est ordinairement intervenue pour indemniser les habitants victimes de catastrophes dites naturelles. Sur la base des procès-verbaux de dégâts, attestés par les autorités royales, les habitants pouvaient obtenir une diminution de leur charge fiscale. Cette procédure était cependant plus ou moins régulière et différemment formalisée selon les provinces. On peut alors légitimement se demander si le témoignage n'a pas volontairement augmenté les dommages. De fait, «les enquêtes conduisent notamment à relativiser les descriptions exagérées des sinistres qui servent souvent à justifier des demandes appuyées de dégrèvements fiscaux » (Favier et Granet-Abisset, 2000).

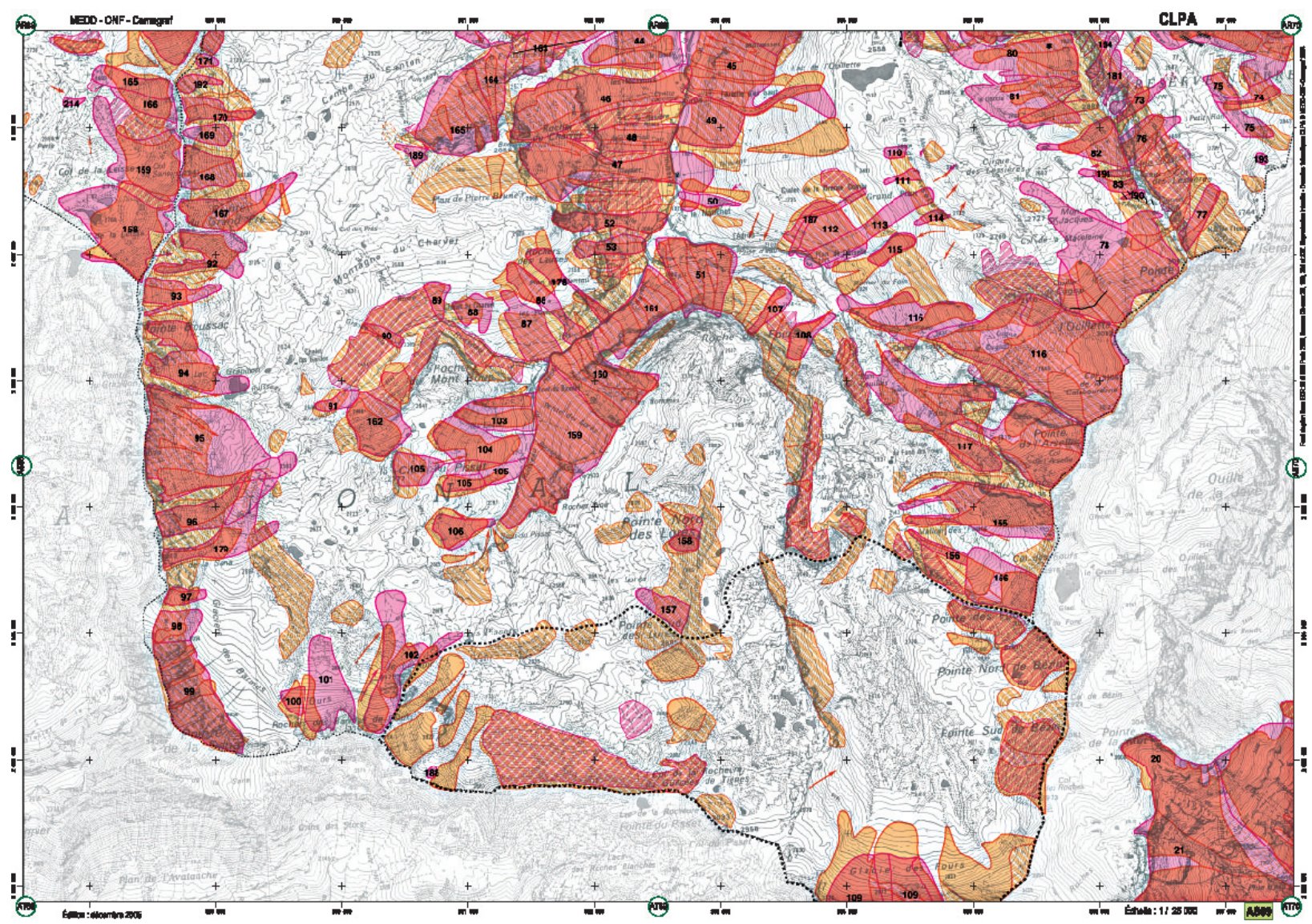

Figure 1 - CLPA AS69 Haute-Maurienne. 
Contrairement aux attentes, on observe que le caractère hétérogène des informations EPA diminue la crédibilité des calculs de probabilité issus de la base de données. Il ne s'agit pas que d'imprécision d'information, mais d'information en relation avec des peuplements et des milieux physiques qui ne correspondent plus aux actuels. L'imprécision et l'incertitude ne sont pas synonymes. Cela permet de recouper et de justifier la deuxième source d'incertitude que mentionnent Godard et al., (2002).

\section{2 - Seconde source d'incertitude : l'«impossibilité d'établir des probabilités face à une situation nouvelle»}

(Godard et al., 2002)

Le caractère nouveau est directement perceptible par les mutations des peuplements montagnards, qui concernent le bâti ou les ouvrages de protection. Il est également associé aux mutations environnementales. Elles sont beaucoup moins facilement décelables, mais même la bibliographie qui ne partage pas les conclusions actuelles du groupe international des experts sur le changement climatique (GIEC) peut les admettre (Leroux, 2002).

\section{a - Mutations climatiques et impacts sur les risques d'avalanches}

Le réchauffement climatique sur lequel travaillent les experts du GIEC entraînera vraisemblablement une forte diminution de la durée de l'enneigement, et des phénomènes d'avalanche, dans les zones de moyenne montagne, actuellement déjà relativement peu concernées par ces phénomènes, et avec des enjeux moindres. En revanche, rien dans les évolutions annoncées ne permet de prévoir une diminution des aléas, et des enjeux, dans les zones d'altitude qui sont déjà actuellement les plus sensibles. Mais le fond du problème est lié au caractère difficilement prévisible des relations entre les facteurs de forçage climatique (les Gaz à Effets de Serre essentiellement) et l'évolution des précipitations comme des températures, en raison de la multiplicité des interactions partielles entre les paramètres climatiques (Beltrando, 2004). C'est la principale raison pour laquelle sont développés des scénarios d'évolution, dont les résultats varient d'ailleurs en fonction des paramètres entrés dans les modèles (Menoni, 1997). Les mutations climatiques actuellement étudiées par les chercheurs révèlent donc des surprises, ce qui justifie le recours à l'incertitude.

Ce d'autant plus que, dans le même temps, on observe :

\section{b - L'intensification des travaux de protection et de l'urbanisation}

Cette tendance de fond, qui connaît des exceptions locales, s'identifie très clairement sur les terrains étudiés. Du moins là où les enjeux socialement et politiquement reconnus comme élevés peuvent justifier la multiplication des travaux. Sur le fond, les travaux de correction permettent de réduire les fréquences des avalanches dommageables, ce qui est d'ailleurs clairement l'un des objectifs poursuivis. Une des contreparties est liée au fait que ces travaux modifient forcément les avalanches, par exemple leurs trajectoires pour les avalanches de fond. De ce fait, et automatiquement, toujours plus d'ouvrages démultiplie les situations nouvelles, pour lesquelles les calculs de probabilité s'appuyant sur les événements passés sont plus difficiles voire douteux.

Il en va de même pour les enjeux, puisque la réalisation des travaux de correction favorise l'urbanisation sur les secteurs à fréquence d'événement moindre, par exemple dans les zones bleues des PPR. Favorise, car elle n'est pas systématique. On observe par exemple la lenteur d'évolution du bâti dans la zone bleue du PPR de Chamonix qui correspond à la partie terminale du couloir de Taconnaz. Ceci est facilité par le bilan qui avait été dressé à la fin de la décennie 1990 (Pigeon, 1998).

On devine alors ce qui pousse les chercheurs et les gestionnaires à prendre en compte l'incertitude comme à essayer de la réduire. En effet, une troisième approche de l'incertitude porte sur «l'incapacité à déterminer une liste complète de résultats possibles de l'action» (Godard, 2002). Elle a été tout particulièrement révélée par les avalanches de 1999, qui ont suscité une remise en cause de l'EPA comme des CLPA en France. Elle est confirmée par la bibliographie qui accompagne cet article (Lambert, 2004).

\section{II - Pourquoi la reconnaissance aCCRue de L’incertitude liée aux aValanches ?}

On peut la mettre en relation étroite avec l'intensification contemporaine de l'urbanisation alpine. Elle nécessite un accompagnement, la prise en compte du risque d'avalanche. Or, l'expérience en retour montre que la gestion par les risques connaît des limites, et que la prévisibilité demeure très partielle. D'où des réponses politiques qui cherchent à réduire l'incertitude dans des conditions qui soient politiquement et socialement acceptables. 


\section{1 - L'intensification de l'urbanisation alpine incite à mesurer toujours plus le risque d'avalanches}

L'intensification de l'urbanisation signifie une augmentation de la mobilité, en particulier routière, et une tendance à la densification et/ou à l'extension du bâti. Dans les deux cas, la CLPA et l'EPA sont mobilisées, afin d'accompagner ces évolutions.

$\mathrm{Si}$ actuellement la construction de routes nouvelles est peu fréquente, le maintien d'accès hivernaux en altitude, auparavant non déneigés, est exigé de plus en plus. Les fermetures temporaires lors d'importantes chutes de neige sont de moins en moins tolérées et justifient, dans un second temps, une demande d'équipements de protection. La CLPA et l'EPA constituent des outils de base, pour la définition, la gestion des ouvrages (routiers par exemple) et l'établissement des priorités. Elles fournissent des données utiles à la conception et à la gestion des voies d'accès. Pour toutes ces utilisations, dépassant leur finalité initiale, les données EPA et CLPA sont utilisées parce qu'elles sont les plus objectives, en matière d'avalanche. Les $2 / 3$ des couloirs observés à l'EPA concernent des voies de communication (IGE, 2006)

L'accessibilité accrue contribue à augmenter la pression sur un foncier constructible rare en montagne. Sa valeur est liée aux enjeux présents sur le territoire (donc à l'économie, par exemple les activités de loisirs : sports d'hiver, et activités de sports de montagne et de nature en été). La population du massif alpin français s'est accrue de 950000 personnes en 40 ans ; soit plus de $1 \%$ par an pendant les 10 dernières années, alors que la moyenne en France se situe à 0,37\% par an (IGE, 2006, p30). Perlik (2002) rappelle que «la croissance des zones urbaines dans l'ensemble est, dans les Alpes, presque complètement due à la croissance des communes en couronne». La périurbanisation rapproche la population alpine des massifs élevés, sujets à l'aléa avalanche.

Cette évolution de l'économie de l'espace montagnard conduit à une recherche de terrains constructibles ou à densifier l'urbanisation existante. La nécessité de réduire les incertitudes liées aux bases de données concernant les avalanches est confirmée par le toilettage de l'EPA. Une convention conclue pour la période 2002-2006 visait notamment à améliorer la procédure de collecte des données, la valorisation et la révision des sites observés. Il s'agissait de redéployer la collecte d'informations vers les zones à enjeux d'urbanisme actuels ou potentiels (IGE, 2006) : une des recommandations émises par l'Inspection Générale de l'Environnement lors du retour d'expérience sur l'avalanche du 9 février 1999 à Montroc. Cette problématique n'est pas spécifique à la France, nous la retrouvons avec les expertises menées en Suisse à la suite de l'hiver 1999.

\section{2 - Les avalanches de février 1999 ont révélé l'existence de l'incertitude}

Du 9 au 11 février 1999, sur les 18 avalanches observées dans la vallée de Chamonix, 8 ont dépassé les limites de la CLPA. Cela révèle à la fois la fréquence rare de cet épisode et les choix méthodologiques qui sous-tendent la CLPA, basée sur l'aléa de référence centennal. Le maire avait fait évacuer dans la journée précédant le sinistre et le 9 février au moins 40 personnes des zones menacées (IGE, 2000). Le 9 février 1999, l'avalanche de Montroc s'est déclenchée à $2450 \mathrm{~m}$ d'altitude suite à une rupture de plaque de 1,5 $\mathrm{m}$ d'épaisseur. La vitesse de l'aérosol lui a permis de franchir le replat glaciaire situé à 1900 m, et de mobiliser le stock neigeux disponible sur le versant en contrebas. Comme le précise Lambert (2004), les conditions nivo-météorologiques particulières expliquent essentiellement pourquoi cette avalanche a pu «dépasser les limites habituellement admises ou connues». On estime à $300000 \mathrm{~m}^{3} \mathrm{le}$ volume mis en mouvement, qui a donné un volume de $150000 \mathrm{~m}^{3}$ dans la zone d'arrêt, la hauteur moyenne de l'accumulation étant de 5 mètres. Le bilan a été de 12 morts, 14 chalets ont été détruits, 6 endommagés, tous situés en zones bleue ou blanche du Plan de Prévention des Risques.

Le retour d'expérience a fait ressortir les limites de la cartographie, comme l'importance des facteurs d'incertitude.

De très faibles distances entre des zones à dégâts très forts et celles à dégâts nuls ont été observées. Cela montre les limites de représentation par la cartographie prévisionnelle : à l'échelle actuelle des études, au $1 / 25000$, l'épaisseur du trait laisse une indétermination de $25 \mathrm{~m}$. Les méthodes utilisées sont donc remises en cause (IGE, 2000).

L'épisode neigeux précédant la catastrophe, sur le seul critère de la hauteur de neige, n'atteignait pas les records historiques. Par contre l'événement météorologique dans sa complexité (vent fort, température très basse, durée...) était très rare. Pour Chamonix, les estimations dépassent 100-150 ans pour les périodes de retour (IGE, 2000)

L'ampleur du phénomène, notamment par sa dénivelée $(1000 \mathrm{~m})$, a été exceptionnelle. Il n'est pas lié qu'à des particularités françaises, ce que confirment les retours d'expérience helvétiques (1500 m à Evolène, $1900 \mathrm{~m}$ à Morgex). Les trajectoires y furent aussi mentionnées comme «inhabituelles» (IGE, 2000), déviant par rapport aux trajets connus.

Concernant ce même hiver, l'Institut Fédéral Suisse de Recherche sur la Neige et les Avalanches a étudié les limites et les défauts des procédures et du zonage du risque en Suisse. Il a été noté que $80 \%$ des avalanches se sont produites dans les limites du zonage réglementaire mais $20 \%$ les ont dépassées. Plusieurs déficiences sont été exposées (TR ALPRISK, 2005) : 
- «les distances d'arrêts des avalanches poudreuses ont été sous-estimées,

- l'occurrence d'évènements multiples dans le même couloir a modifié les conditions d'écoulements et donc augmenté les extensions en particuliers latérales ;

- les dépôts anciens en lissant les couloirs ont diminué le frottement et réduit l'efficacité des ouvrages de protection ce qui s'est traduit par une augmentation significative des distances d'arrêts ».
Les acteurs publics ont alors essayé de réduire l'incertitude, tout particulièrement en intégrant les retours d'expérience. Cet hiver 1999 a poussé à admettre les limites des dispositifs antérieurs. Et donc aussi l'incapacité à déterminer une liste complète de résultats possibles de l'action, qui représente une des signatures de l'incertitude selon Godard et al. (2002). Les limites de la prédiction ne signifient toutefois pas l'impossibilité de réduire l'incertitude, d'où les démarches qu'expose le point suivant.

\section{III - COMMENT ESPÉRER RÉDUIRE L'INCERTITUDE ?}

Nous avons pu voir que les nombreux retours d'expérience suite aux avalanches de 1999 ont pointé les limites de la gestion du risque. Ils ont recommandé notamment une meilleure connaissance de l'aléa en insistant sur l'utilisation de la modélisation. Ils ont aussi mis en avant la nécessité de développer une nouvelle génération de PPR comprenant une analyse précise de la vulnérabilité. Par ailleurs, l'IGE a recommandé le développement de SIG pour une gestion des données en veillant à leur pertinence et à leur objectivité.

Ces recommandations représentent autant de moyens visant à réduire l'incertitude, que nous reprenons ci-dessous. Il s'agit de préciser l'information et ses limites, de l'actualiser, donc d'espérer améliorer la définition de l'aléa de référence. Cela permet aussi de prendre en compte et de justifier des marges d'erreur dans la définition des ouvrages de protection comme des zonages fonciers, sans pour autant prétendre à l'élimination des risques. En effet, même dans le cas du recours massif aux solutions structurelles et aux tunnels, les expériences en retour permettent de démontrer que l'incertitude n'a pas disparu. Plus que jamais, le renforcement de la sécurité civile apparaît essentiel.

\section{1 - Préciser la donnée et ses limites pour améliorer la fiabilité du traitement de l'information}

Le CEMAGREF a mis en place des outils de traitement des données permettant de filtrer un certain nombre d'erreurs ponctuelles, d'apprécier la régularité temporelle des observations, et de mettre en évidence les corrélations des occurrences d'événements entre les sites. Pour les données les plus surprenantes, des expertises in situ sont réalisées. La base de données événements du service de Restauration des Terrains en Montagne évalue également le degré de fiabilité de la donnée. La démarche permet de garder une trace des données douteuses pour pouvoir les corriger ultérieurement.

Augmenter la connaissance des informations, et de leurs limites, sur les sites concernés par des avalanches passées permet de donner plus de crédibilité aux outils qui permettent d'actualiser et de modéliser le risque d'avalanches. Il s'agit d'une étape essentielle dans un certain nombre de démarches comme la définition d'un aléa de référence. La modélisation vient en complément de l'historicité des phénomènes mais elle s'appuie également dessus pour caler le modèle retenu. La reconnaissance des limites de l'information est donc essentielle.

En effet, une des utilisations des bases de données EPA et CLPA est d'aider à définir l'aléa de référence dans les PPR. Cette définition est plus compliquée qu'en hydraulique. L'aléa de référence est basé sur l'historique («la plus grande avalanche historique connue») et sur la modélisation. Actuellement, les séries historiques EPA fiables remontent rarement à plus de quelques décennies, la CLPA ne date que d'une trentaine d'année et ne répertorie pas tous les événements antérieurs. La modélisation statistique simple, calée avec des séries historiques, nécessite des séries de données fiables et assez longues. La modélisation déterministe, qui décrit les phénomènes physiques, reste en développement. La réduction du nombre de couloirs observés dans l'EPA (2549 sites abandonnés, 714 créés ; IGE, 2006), qui pourrait être une source d'économie, n'apparaît pas pertinente actuellement, au vu de la faiblesse des corrélations statistiques possibles. En effet, l'analyse statistique du couple intensitérécurrence des plus longues séries, de plus d'un siècle (en Savoie et Haute-Savoie) permet d'approcher l'évènement centennal. L'événement le plus grand observé étant déterminant pour le zonage, l'EPA qui le décrit est irremplaçable. Une dégradation de l'EPA (interruption, ou réduction du nombre de couloirs observés) modifierait cette référence. Pour l'approche de l'avalanche maximale vraisemblable, les données météos de l'EPA contribuent à l'établissement de corrélations locales entre précipitations neigeuses et événements importants. Il est en effet important de pouvoir graduer les mesures de protection (sauvegarde) en fonction de l'évènement prévisible. Jusqu'à présent, Météo France est réticent à passer d'une note de risque par massif, à une prévision par versant. 
Par conséquent, la réduction de l'incertitude passe aussi par des efforts de modélisation, dans le cadre de scénarios. Il s'agit d'intégrer dans le raisonnement l'incertitude spatiale liée au phénomène et, dans une certaine mesure, l'incertitude liée aux choix des paramètres qui sous-tendent les calculs.

On retrouve avec ce dernier point l'accentuation de l'incertitude, notamment par la multiplication des travaux de correction. Une parade revient à prendre en compte les marges d'erreurs qui sont estimées, mais aussi admettre les limites des travaux de correction. Ces démarches reviennent alors à admettre l'incertitude tout en cherchant à l'intégrer à l'effort de gestion.

\section{2 - Prendre en compte des marges d'erreur dans la conception des ouvrages correctifs comme dans les zonages fonciers?}

La première solution a été évoquée à propos des rapports qui ont suivi la crise de 1999. Ils insistent sur le retour d'expérience des pratiques utilisées dans le Val d'Aoste concernant les constructions pouvant être atteintes par les avalanches. Ces prescriptions sont fondées sur les paramètres dynamiques des avalanches. Les calculs comportent un coefficient de sécurité prenant en compte à la fois les incertitudes des hypothèses de départ (aléa de référence) et celles liées aux calculs de génie civil. Un coefficient de sécurité de 1,5 est appliqué à titre de pondération des vulnérabilités structurelles liées à l'habitation, mais aussi à la circulation en ce qui concerne les ouvrages de protection (IGE, 2000).

La reconnaissance de l'incertitude par ces coefficients de sécurité souligne la difficulté de gérer le risque d'avalanche par des cartes juridiquement opposables. Quelle est la légitimité d'un trait séparant deux zones, alors que ce trait a valeur juridique, et contribue à modifier les valeurs foncières ? Cette question se retrouve au cœur de la problématique de l'Avalanche Maximale Vraisemblable (AMV).

Cette notion correspond à la définition d'une zone plus large que celle concernée par l'aléa de référence, centennal ou plus grand connu. La zone d'influence de cet événement rare serait à prendre en compte d'abord pour la sécurité des personnes. C'est ce qu'affirme explicitement la réponse du Ministère de l'Intérieur à la question écrite $\mathrm{n}^{\circ} 01368$ de Monsieur Marcel Rainaud, le 26 mars 2009 (Minsitère de l'Intérieur et Sénat, 2009) En effet, les PPR gèrent l'urbanisation actuelle. Mais demeurent les problèmes que posent les bâtiments qui leur préexistent, comme ceux des zones habitées où il existe un risque en cas de phénomène avalancheux exceptionnel.
Indépendamment du débat induit sur les responsabilités, l'approche actuelle de détermination des zones « jaunes » concernées par cet aléa rare est donc définie par la topographie et les caractéristiques de la zone d'arrêt. Il ne paraît pas possible actuellement d'apporter une solution autre qu'experte. En effet, on ne dispose pas des méthodes de l'hydraulique pour extrapoler de façon vraisemblable une avalanche de temps de retour très long à partir de séries de quelques décennies. L'approche statistique paraît donc être illusoire pour définir cette délimitation. D'autre part, la modélisation déterministe qui permettra de représenter un événement de grande ampleur en décrivant les phénomènes physiques en jeu demande encore à être calée.

On en vient donc à reconnaître que les travaux de correction, tout comme les zonages fonciers, ne parviennent pas à éliminer l'incertitude. Il s'agit néanmoins de la réduire. C'est d'ailleurs dans ce sens qu'évolue le dispositif français, la loi de modernisation de la sécurité civile permettant de prendre acte des limites des efforts de gestion antérieurs. En effet, l'expérience en retour montre l'impossibilité d'éliminer les risques quels que soient les investissements dans les travaux de correction envisagés.

\section{3 - L'impossibilité d'éliminer l'incertitude malgré de lourds investissements... d'où la nécessité de renforcer la sécurité civile}

Tignes présente de très importants enjeux liés aux conditions d'accès de sa station (30 000 lits touristiques). Ses deux routes départementales (902 et 87 ) sont très exposées aux risques d'avalanches. Cette commune s'inscrit dans le cadre d'une politique préventive intensive émanant du Conseil Général de Savoie. $92 \%$ des sommes investies dans les travaux de protection contre les risques sont des travaux liés à la protection contre le risque avalanche (tableau 1). Ces investissements ont été décuplés avec le plan qualité route (2000-2006) : pose de râteliers, de filets pare-neige, constructions de galeries paravalanches... (figure 2). Les 8 kilomètres de la RD87 sont exposés à 20 couloirs d'avalanche et sont empruntés quotidiennement par plus de 7000 véhicules en hiver (figure 3 ). La totalité de son parcours est

\begin{tabular}{|l|c|c|}
\hline \multicolumn{1}{|c|}{ risque } & montant investi en $€$ & $\%$ \\
\hline avalanche & $4794937 €$ & 90,8 \\
\hline chute de blocs, pierres & $221959 €$ & 4,2 \\
\hline éboulement, écourlement & $16221 €$ & 0,3 \\
\hline affaissement, effondrement & $76225 €$ & 1,4 \\
\hline inondation & $19818 €$ & 0,4 \\
\hline laves torrentielles & $152445 €$ & 2,9 \\
\hline
\end{tabular}

Tableau 1 - Montant annuel de travaux de protection réalisés contre les risques « naturels » sur les 2 routes départementales d'accès à Tignes, de 1993 à 2003. 


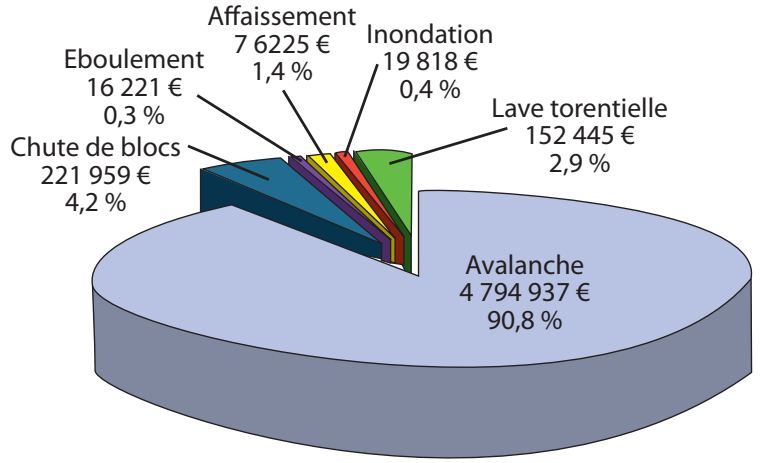

Figure 2 - Représentation graphique des investissements sur la commune de Tignes de 1993 à 2003.

depuis peu sécurisée, notamment grâce à la réalisation d'une déviation au niveau des Boisses, ancien dernier point noir du tronçon. Cela a nécessité des investissements à hauteur de 1,8 million d'euros (2006).

Toutefois, couvrir les réseaux routiers revient à déplacer l'incertitude comme le confirment les catastrophes associées aux tunnels (Tunnel du Mont-Blanc, par exemple, 1999, Le Griel, 2001). C'est également démontrable à partir du franchissement du col du Gothard (Pigeon, 2004). Sur le Gothard, la solution tunnel a permis de réduire considérablement les dommages liés aux avalanches, et notamment ceux, indi- rects, associés aux périodes de fermeture préventive de la route. Mais, comme en France, cette solution a favorisé les dommages liés aux incendies dans les tunnels. En fait, on pourrait prétendre que l'évolution reproduit les effets non désirés liés aux ouvrages classiques corrigeant les avalanches. Ils réduisent les fréquences, tout en contribuant à préparer de futures catastrophes, événements de fréquence rare, et d'intensité supérieure. Là encore, l'évolution n'est toutefois que partiellement prévisible, et partiellement quantifiable. Ce malgré le recours à un mode de représentation d'aspect quantitatif, et développé à l'origine par un ingénieur travaillant sur le risque nucléaire, la courbe de Farmer (Pigeon, 2005).

Les limites des solutions structurelles justifient alors le renforcement de la sécurité civile, comme l'accompagnement, souvent politiquement délicat, de l'urbanisation par le zonage foncier. Ce sont précisément les solutions que développe la France. Si l'Etat est seul compétent pour « dire » le risque, notamment à travers la procédure PPR, dont la prescription et l'approbation sont de la compétence du préfet, les compétences et les responsabilités en matière de protection de la population (documents d'organisation de la sécurité, Plan Communal de Sauvegarde par exemple s'articulant avec les plans ORSEC) et en matière d'information préventive incombent aux collectivités locales. La loi Risques de juillet 2003 appelé loi Bachelot l'a rappelé.

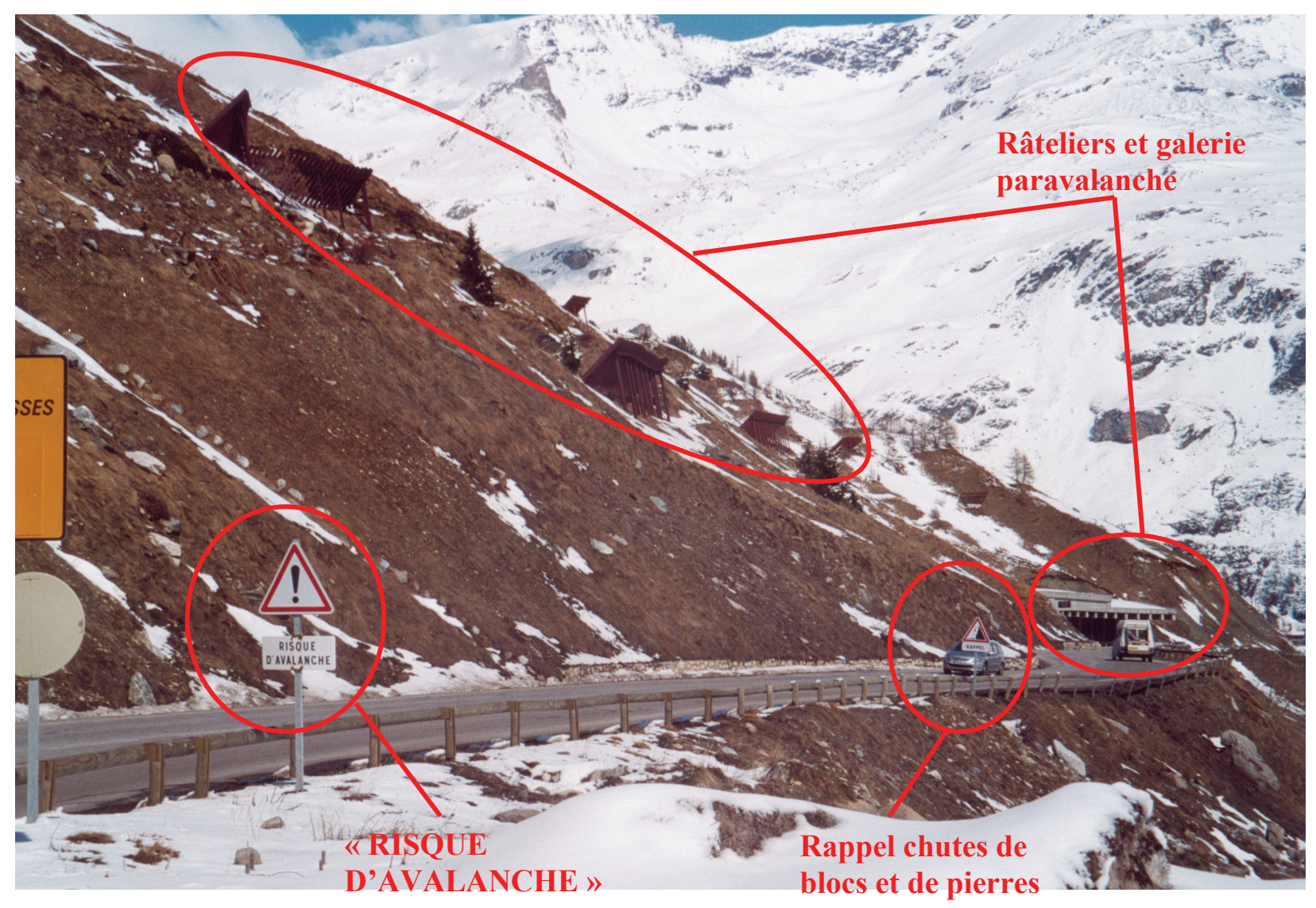

Figure 3 - Marques territoriales du risque sur la RD87 entre Les Boisses et Tignes Le Lac. Cliché, A. Moulin, 11 mars 2004. 
Cette situation engendre une demande de plus en plus forte de la part des élus en matière de prévention et de prévision. Les maires sont ainsi à la recherche d'outils, de méthodes pour gérer et protéger leurs enjeux. Ils souhaitent que l'essentiel de leur territoire reste ouvert à la construction.

\section{Conclusion}

L'article permet de comprendre pourquoi l'incertitude ne peut plus être considérée comme secondaire dans les efforts de compréhension et de gestion des risques concernant les avalanches aujourd'hui. Du reste, cette problématique dépasse les risques liés aux avalanches. Il s'agit d'un problème majeur, qui implique l'Humanité. Il est en relation directe avec l'intensification mondiale de l'urbanisation, comme avec les mutations environnementales qui l'accompagnent. Les travaux du GIEC, qui visent aussi à réduire l'incertitude, confirment cette tendance de fond. L'un des problèmes majeurs est lié à la reconnaissance de situations nouvelles, qu'elles concernent les peuplements humains ou les mutations climatiques. Elles réduisent considérablement la crédibilité des enseignements tirés du passé, comme des calculs de probabilités portant sur des populations statistiques à hétérogénéité croissante. $\mathrm{Du}$ reste, c'est ce que vivent concrètement les assureurs, qui observent des écarts importants entre ce que prédisent les modèles et les retours d'expérience vécus (Domenichini, 2008).

Dans le même temps, l'augmentation des enjeux fait que les sociétés urbanisées n'ont d'autres choix que de chercher à réduire l'incertitude et/ou de l'intégrer dans les politiques visant à piloter l'urbanisation. En effet, ne pas le faire revient à favoriser de futures catastrophes. Les événements de fréquence rare mais d'intensité élevée, comme ceux de l'hiver 1999, attirent à la fois l'attention sur la réalité du processus et sur la volonté politique de le contrer le plus possible. Les efforts pour tester la validité de l'EPA et des CLPA en France, après 1999, prouvent clairement la reconnaissance de l'incertitude comme la volonté, la nécessité de la réduire. On remarquera au passage que cet effort politique est tout à fait compatible avec les principes du développement durable. Ces derniers posent la nécessité de renforcer la résilience des sociétés locales, soit leur capacité à conserver leurs structures fondamentales en cas d'événement partiellement prévisible. Le renforcement de la sécurité civile, un moyen de réduire l'incertitude, contribue clairement à cette évolution. Il a été pris en compte très rapidement par des communes particulièrement exposées, comme celle de Chamonix (Garcin-Parraguez, 2004). Ce n'est pas un hasard si plusieurs programmes de recherche internationaux sont impliqués dans cette voie.

Toutefois, l'évolution suppose aussi d'admettre l'impossibilité d'éliminer l'incertitude. Ce point pose particulièrement problème, surtout dès lors que des enjeux majeurs, notamment liés au foncier, sont présents. On touche ici du doigt les difficultés que connaissent les documents comme les PPR, qui, une fois approuvés par arrêté préfectoral, sont opposables aux tiers, et contribuent à modifier les valeurs foncières. Or, à la lumière de ce qui précède, on voit que la justification du trait à valeur juridique ne pourra jamais être autre que partielle, et, partant, encore plus susceptible d'être contestée, quels que soient l'expert et son travail.

Enfin, il nous paraît utile d'attirer l'attention sur un problème de fond, qui est lié aux relations entre urbanisation et incertitude. Il ressort des différents retours d'expérience l'impossibilité d'éliminer l'incertitude, ici liée aux avalanches. Mais aussi la nécessité de la réduire afin de pouvoir davantage justifier l'indispensable gestion des risques. On peut alors identifier un processus majeur. La tendance fondamentale est bien celle qui consiste, grâce aux travaux de correction, à troquer des événements de fréquence élevée et défavorables à l'urbanisation, pour des événements de fréquence rare et potentiellement catastrophiques, mais qui ne défavorisent pas la poursuite actuelle de l'urbanisation. Il s'agit désormais d'accompagner cette tendance de fond, en réduisant préventivement, et le plus possible, les dommages en cas de phénomènes de fréquence rare, excédant les possibilités des travaux de correction. Ces coévolutions urbanisation-incertitude, de tendance positive au sens systémique, confirment l'intérêt interprétatif des théories de la complexité.

\section{BibLIOGRAPHIE}

ANCEY C., 2001. Analyse fréquentielle du risque d'avalanche : quel enjeu? Neige et avalanches, 94, 12-16.

Beltrando G., 2004. Les climats. A. Colin, Paris, 261 p.

BRUgnot G., 2002. La cartographie des avalanches. Neige et avalanche, 97,14-19.
Cabos S., 2003. Note exploratoire sur le nombre de constructions situées dans les emprises d'avalanches CLPA en France. Stage maîtrise IGA, CEMAGREF, 11 p.

CEMAGREF, 2003. Corrélation entre les événements et les sites avalancheux. Rapport inédit, 24 p. 
Domenichini J., 2008. Calcul de la sinistralité relative à des aléas «naturels » et anthropiques rares : systèmes d'information géographique (SIG), retour d'expérience et méthodes prospectives pour l'évaluation de sinistres inondation et terrorisme en réassurance. Thèse Université Paris I, $255 \mathrm{p}$.

Dupont C., Pigeon P., 2008. Le Haut-Rhône et son bassinversant montagneux : pour une gestion intégrée des territoires transfrontaliers. Institut de la Montagne, Chambéry, $117 \mathrm{p}$.

Dupuy J.-P., 2002. Pour un catastrophisme éclairé. Le Seuil, Paris, $216 \mathrm{p}$

Favier R., Granet-Abisset A.-M., 2000. Pour une histoire des catastrophes naturelles : une étape dans une recherche collective. In Histoire et mémoire des risques naturels. MSH Alpes, Grenoble, 271-276.

GarCia S., 2002. Analyse de la régularité des observations de l'Enquête Permanente sur les Avalanches. CEMAGREF, $45 \mathrm{p}$.

Garcin-Parraguez A., 2004. Plan d'alerte et d'évacuation, risques d'avalanches à Chamonix. Rap. inéd. Master 2, Univ. Lyon III, 103 p.

Godard O., Henry C., Lagadec P., Michel-Kerjan E., 2002. Traité des nouveaux risques. Gallimard, Paris : 620 p.

IFEN (Institut Français DE L'ENVIRONNEMENT), 1998. Acquis et lacunes de l'information statistique sur l'environnement. Coll. Notes de méthode, $74 \mathrm{p}$.

IGE (INSPECTION GÉNÉRALE DE L'ENVIRONNEMENT), 2000. Retour d'expérience sur l'avalanche du 9 février 1999 à Montroc, commune de Chamonix après la phase contradictoire. $69 \mathrm{p}$.

Jamard A.L., Garcia S., 2002. L'Enquête Permanente sur les avalanches (EPA) - Statistique descriptive générale des événements et des sites. CEMAGREF, Université Joseph Fourier, $101 \mathrm{p}$.

LAMBERT R., 2004. La gestion spatiale du risque d'avalanche : une question d'actualité. Actes du colloque interpraevent. Site internet: www.expertavalanche.com

LE GRIEL A., 2001. Les organisations territoriales et l'engrenage des risques : réflexions géographiques à partir de l'exemple de l'incendie du tunnel du Mont-Blanc. Actes du colloque de Lyon, «risques et territoires», ENTPE / CNRS, 1, 103-116.
LERoux M., 2002. «Global warming» : mythe ou réalité? L'évolution réelle de la dynamique du temps. Ann. Géogr., 624, 115-137.

MANCHE Y., 2000. Analyse spatiale et mise en place de systèmes d'information pour l'évaluation de la vulnérabilité des territoires de montagne face aux risques naturels. Thèse Université Joseph Fourier, $172 \mathrm{p}$.

MEDD (Ministère de L'ENVIRONNEMENT ET DU DÉVELOPPEMENT Durable), 2006. Rapport de l'IGE, Poursuite du programme d'observation des avalanches. $56 \mathrm{p}$.

Menoni S., 1997. Pianificazione e incertezza. Elementi per la valutazione e la gestione dei rischi territoriali. Franco Angeli éd., Milan, 256 p.

Ministère de L'Intérieur et SÉnat. 2009. Prise en compte du risque tricentennal dans la cartographie des Plans de prévention des risques naturels majeurs. Question écrite $\mathrm{n}^{\circ} 01368$ de $\mathrm{M}$. Marcel Rainaud et réponse du Ministère de l'Intérieur. JO Sénat, 19 mars 2009, p. 710

Perlik M., 2002. Une typologie fonctionnelle des zones urbaines en vue d'une analyse des processus d'urbanisation et des processus économiques dans les Alpes. In Grange, D.J. L'espace alpin et la modernité. P.U.G., Grenoble. 504 p., 167-175.

Pigeon P., 1998. Représentation cartographique du risque et vulnérabilité liée à la pression foncière touristique (Taconnaz, Les Houches et Vers-Le-Nant, Chamonix). Rev. Géogr. Alp., 86(2), 101-113.

Pigeon P., 2004. Gestion des risques liés au franchissement alpin et peuplements helvétiques : l'exemple du Gothard. Actes du colloque «Traverser les Montagnes», Chambéry 3et 4 octobre 2002. Collection EDYTEM, 2, Cahiers de Géographie, 151-160.

PigeOn P., 2005. Géographie critique des risques. EconomicaAnthropos, Paris, $280 \mathrm{p}$.

Strazzeri D., Manche., Y. 1998. L'enquête permanente sur les avalanches. Rev. Géogr. Alp., 86(2), 45-52.

TR ALPRISK, 2004. Référentiel pluriannuel d'objectif 20042008. Rapp. inéd. Département «Ressources en eau, usages et risques». $26 \mathrm{p}$. 



\section{NEIGE et GLACE de MONTAGNE \\ Reconstitution, dynamique, pratiques}

\section{Sommaire}

Editorial

Introduction

\section{1 - Reconstitution}

Coutterand S. et al. - Le lobe glaciaire lyonnais au maximum würmien : glacier du Rhône ou/et glaciers savoyards ?

Ravanel L. et al. - Désenglacement du haut bassin versant du Vorz (massif de Belledonne, Isère), au Tardiglaciaire et à l'Holocène.

Rey P.-J. - Sociétés et fluctuations du climat dans les Alpes nord-occidentales au Néolithique moyen.

Le Roy et al. - Étude des fluctuations glaciaires du Petit Âge de Glace dans le Massif des Écrins : apports de la lichénometrie.

Kirkbride M.P. - Datation des moraines holocènes d'Islande par tephrochronologie : un état de l'art.

Le Roy et al. - La dendroglaciologie, ou l'apport de l'étude des cernes d'arbres pour la reconstitution des fluctuations glaciaires holocènes.

Rabatel A. - Évolution glaciaire dans les andes subtropicales chiliennes entre 1955 et 2007 : conséquences pour la ressource en eau.

Le Roy et al. - Un inventaire des aérophotographies du massif du Mont Blanc.

\section{2 - Dynamique}

Ravanel L. - Évolution géomorphologique de la haute montagne alpine dans le contexte actuel de réchauffement climatique.

Gruber S. - Le permafrost de haute montagne.

Deline P. et al. - L'Aiguille du Midi (massif du Mont Blanc) : un site remarquable pour l'étude du permafrost des parois d'altitude.

Saulnier G.-M. et al. - Un éléphant volant est-il un oiseau ? Perspectives pour l'observation hydrométéorologique des milieux de montagne.

Jobard S. - L'instrumentation du glacier du Baounet : quels apports pour la traçabilité des mesures environnementales ?

Moreau L. - L'exploration du cryokarst glaciaire et son intérêt scientifique pour l'étude du drainage des eaux de fonte.

Mazué R. et al. - Suivi de l'évolution de la couverture detritique d'un glacier noir par photo-comparaison : le glacier d'Estelette.

\section{3 - Pratiques}

Paccard P. - Réchauffement climatique et ressource neige en domaines skiables.

Gauchon C. - Les hivers sans neige et l'économie des sports d'hiver : un phénomène récurrent, une problématique toujours renouvelée.

Laslaz L. - L'exclusion des glaciers des zones centrales des Parcs nationaux de la Vanoise et des Écrins et leur équipement pour le ski d'été.

Cayla N. - Les sentiers d'interprétation glaciaire : des outils de valorisation différenciée des glaciers et de leur territoire.

Lambert R. - Cartozonage : de la carte au zonage du risque avalanche.

Moulin A. et al. - L'incertitude liée aux avalanches dans les Alpes du Nord : identifications et implications pour la gestion.

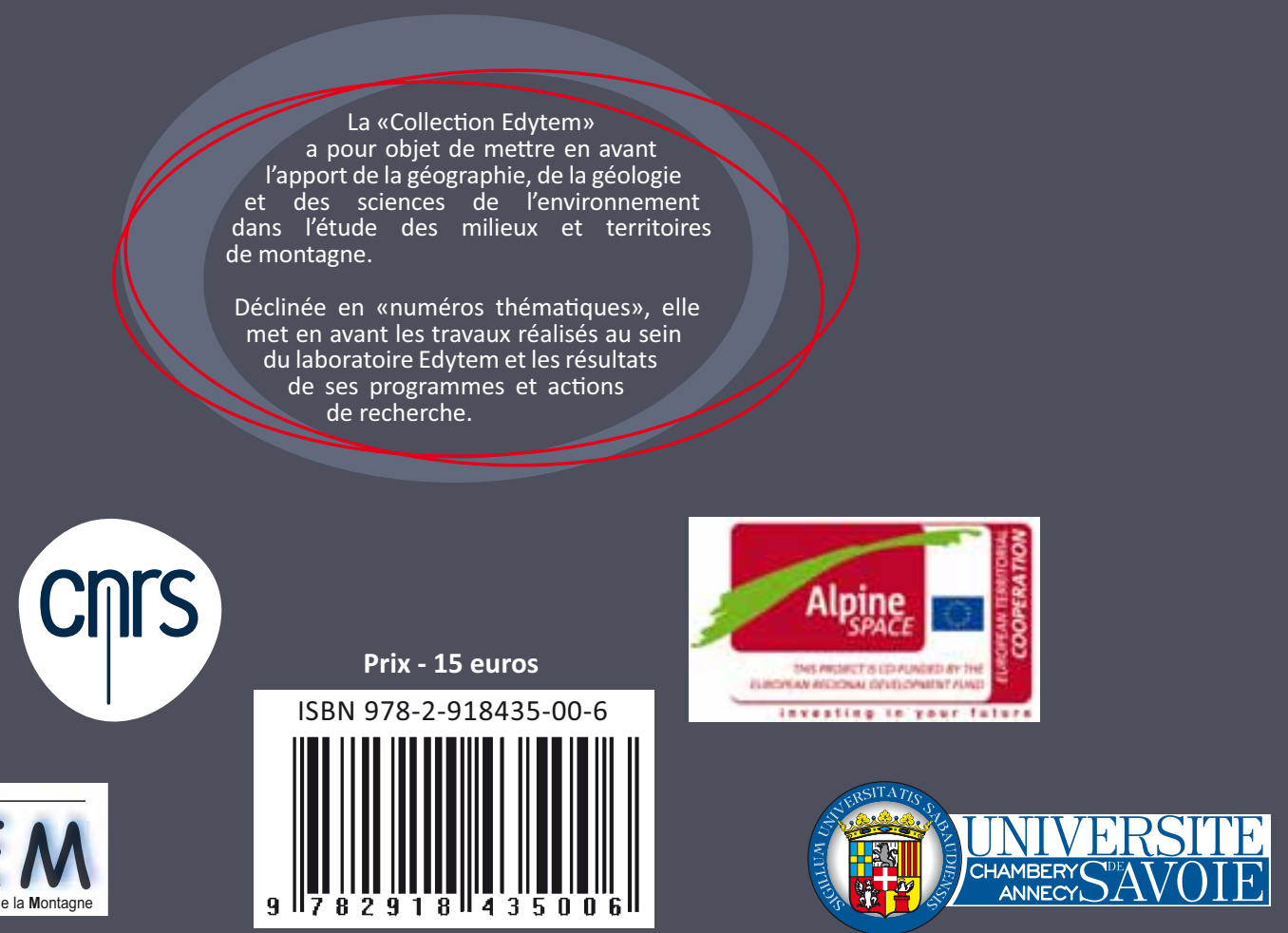

